\title{
Numerical analysis of the Burgers' equation in the presence of uncertainty
}

\author{
Per Pettersson, Gianluca Iaccarino and Jan Nordström \\ Journal Article
}

\section{Tweet}

N.B.: When citing this work, cite the original article.

Original Publication:

Per Pettersson, Gianluca Iaccarino and Jan Nordström, Numerical analysis of the Burgers' equation in the presence of uncertainty, Journal of Computational Physics, 2009. 228(), pp.8394-8412.

http://dx.doi.org/10.1016/j.jcp.2009.08.012

Copyright: Elsevier

http://www.elsevier.com/

Preprint available at: Linköping University Electronic Press

http://urn.kb.se/resolve?urn=urn:nbn:se:liu:diva-68604

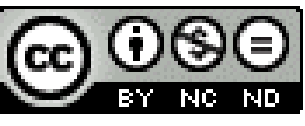




\title{
Numerical analysis of the Burgers' equation in the presence of uncertainty
}

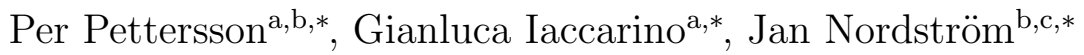 \\ ${ }^{a}$ Department of Mechanical Engineering, Stanford University, Stanford, CA 94305, USA \\ ${ }^{b}$ Department of Information Technology, Uppsala University, SE-75105, Uppsala, Sweden \\ ${ }^{c}$ Department of Aeronautics and Systems Integration, FOI, The Swedish Defense \\ Research Agency, SE-16490 Stockholm, Sweden
}

\section{Abstract}

The Burgers' equation with uncertain initial and boundary conditions is investigated using a Polynomial Chaos (PC) expansion approach where the solution is represented as a truncated series of stochastic, orthogonal polynomials.

The analysis of well posedness for the system resulting after Galerkin projection is presented and follows the pattern of the corresponding deterministic Burgers equation. The numerical discretization is based on spatial derivative operators satisfying the summation by parts property and weak boundary conditions to ensure stability. Similarly to the deterministic case, the explicit time step for the hyperbolic stochastic problem is proportional to the inverse of the largest eigenvalue of the system matrix. The time step naturally decreases compared to the deterministic case since the spectral radius of the continuous problem grows with the number of polynomial chaos

\footnotetext{
*Corresponding author. Tel: 16508628295.

Email addresses: massperp@stanford.edu (Per Pettersson), jops@stanford.edu (Gianluca Iaccarino), jan.nordstrom@foi.se (Jan Nordström)
} 
coefficients. An estimate of the eigenvalues is provided.

A characteristic analysis of the truncated PC system is presented and gives a qualitative description of the development of the system over time for different initial and boundary conditions. It is shown that a precise statistical characterization of the input uncertainty is required and partial information, e.g. the expected values and the variance, are not sufficient to obtain a solution. An analytical solution is derived and the coefficients of the infinite PC expansion are shown to be smooth, while the corresponding coefficients of the truncated expansion are discontinuous.

Keywords: Uncertainty quantification, hyperbolic problems, polynomial chaos, numerical stability

\section{Introduction}

In many physical problems our knowledge is limited by our ability to measure, our bias in the observations and, in general, by an incomplete understanding of the physical processes. When we attempt to simulate the problem numerically, we must account for those limitations, and in addition we must identify the possible limitations of the numerical techniques and phenomenological models employed. In a general sense, we distinguish between errors and uncertainty simply by saying that errors are recognizable deficiencies not due to lack of knowledge, whereas uncertainties are potential and directly related to lack of knowledge [1]. This definition clearly identifies errors as deterministic quantities and uncertainties as stochastic in nature; uncertainty estimation is, therefore, typically treated within a probabilistic framework. 
Numerical simulations are subject to uncertainty in boundary or initial conditions, model parameter values and even in the geometry of the physical domain of the problem (input uncertainty); this results in uncertainty in the output data that must be clearly identified and quantified. Uncertainty quantification is also a fundamental step towards validation and certification of numerical methods to be used for critical decisions. Fields of application of uncertainty quantification include but are not limited to turbulence, climatology [20], turbulent combustion [21], flow in porous media [8], fluid mixing [31] and computational electromagnetics [6].

An example of the need for uncertainty quantification in applications related to methods and problems studied here is the investigation of the aerodynamic stability properties of an airfoil. Uncertainty in physical parameters such as structural frequency and initial pitch angle, affect the probability of limit cycle oscillations. One approach in particular, the polynomial chaos method, has been used to obtain a statistical characterization of the stability limits and to calculate the risk for system failure [27, 2]; this approach will be studied in detail.

There are several approaches to propagate the input uncertainty in numerical simulations; the simplest one is the Monte Carlo method where a vast number of simulations are performed to compute the output statistics. Conversely in the polynomial chaos approach, the solution is expressed as a truncated series and only one simulation is performed. The dimension of the resulting system of equations grows with the number of the terms retained in the series (the order of the polynomial chaos expansion) and the dimension of the stochastic input. 
An increased number of Monte Carlo simulations implies a solution with better converged statistics; on the other hand, in the polynomial chaos approach, one single simulation is sufficient to obtain a complete statistical characterization of the solution. However, the accuracy of this solution is dependent on the order of polynomials considered, and therefore on the truncation in the $\mathrm{PC}$ expansion. Also, convergence requires the solution to be smooth with respect to the parameters describing the input uncertainty [25].

Although of limited practical use in fluid mechanics applications, the Burgers' equation is an interesting and highly non-linear model problem and many results can be extended to other hyperbolic systems, such as the Euler equations. In this paper, a detailed uncertainty quantification analysis is performed for the Burgers' equation; we employ a spectral representation of the solution in the form of polynomial chaos expansion. The equation is stochastic as a result of the uncertainty in the initial and boundary values. Galerkin projection results in a coupled, deterministic system of hyperbolic equations from which the expected values and variance of the solution can be determined.

Previous uncertainty analyses have been performed on the location of the transition layer of a shock discontinuity arising in simulations of the Burgers' equation with non-zero viscosity. Small one-sided perturbations imply large variation in the location of the transition layer, so-called supersensitivity [30], which is a problem in deterministic as well as stochastic simulations. The results from the polynomial chaos approach were accurate and the method was faster than the Monte Carlo method [29, 30]. Burgers' equation with a stochastic forcing term has also been investigated and compared to standard 
Monte Carlo methods [14].

In this work we perform a fundamental analysis of the Burgers' equation and develop a numerical framework to study the effect of uncertainty of the boundary conditions. By assuming that the uncertainty of the boundary data has a Gaussian distribution we allow the occurence of unbounded solutions. Assuming that the boundary data resemble the Gaussian distribution but are bounded to a sufficiently large range does not alter the numerical results. Another reason for allowing unbounded parameter range of the stochastic variable is that the problem becomes more interesting from a mathematical point of view. Convergence is proved by a suitable choice of functional space.

In order to ensure stability of the discretized system of equations, summation by parts operators and weak imposition of boundary conditions $[18,19,5]$ are used to obtain energy estimates. The system is expressed in a split form that combines the conservative and non-conservative formulation [17]. A particular set of artificial dissipation operators [16] and the simultaneous approximation term (SAT) technique [4] for boundary treatment are used to enhance the stability close to the shock. The discretization method is based on a fourth order central difference operator in space and the fourth order Runge-Kutta method in time. The summation by parts operators ensure stable solutions but the allowed time step decreases with increasing PC expansion as a result of the eigenvalues growing with the order of the polynomial order (i.e. the size of the system).

An analytical solution is derived for a discontinuous and uncertain initial condition: the expectation and variance of the solution are shown to be smooth functions while the coefficient of truncated polynomial chaos expan- 
sions are discontinuous. An analysis of the characteristics of the truncated system also shows that the boundary values are time-dependent and suggest a way of imposing accurate boundary conditions.

\section{Polynomial chaos expansion}

The theoretical foundation underlying polynomial chaos was first formulated in [26]. The solution to a partial differential equation characterized by input uncertainty (for simplicity we consider only one uncertain parameter, $\xi$, defined on the probability space $\Omega_{\text {prob }}$ ) is expressed as a spectral expansion:

$$
u(x, t, \xi)=\sum_{i=0}^{\infty} u_{i}(x, t) \Psi_{i}(\xi)
$$

with the inner product

$$
\langle u, v\rangle=\int_{\Omega_{\mathrm{prob}}} u v f(\xi) d \xi
$$

where $f(\xi)$ is the probability density function. The solution is subject to the constraint

$$
\|u\|^{2}=\int_{\Omega_{\mathrm{prob}}} u^{2} f(\xi) d \xi<\infty
$$

i.e. it is a second-order random field (finite variance).

The expected value $E(u)$ and variance $\operatorname{Var}(u)$ can be expressed as functions of the polynomial chaos coefficients. We have

$E(u)=\int_{\Omega_{\mathrm{prob}}} \sum_{i=0}^{\infty} u_{i} \Psi_{i} f(\xi) d \xi=u_{0} \int_{\Omega_{\mathrm{prob}}} f(\xi) d \xi+\int_{\Omega_{\mathrm{prob}}} \sum_{i=1}^{\infty} u_{i} \Psi_{i} f(\xi) d \xi=u_{0}$,

and

$\sigma^{2}=\operatorname{Var}(u)=E\left[u^{2}\right]-(E[u])^{2}=\int_{\Omega_{\mathrm{prob}}} \sum_{i=0}^{\infty} u_{i}^{2} \Psi_{i}^{2} f(\xi) d \xi-u_{0}^{2}=\sum_{i=1}^{\infty} u_{i}^{2}\left\langle\Psi_{i}^{2}\right\rangle$. 
The basis functions $\Psi_{i}(\xi)$ are orthogonal polynomials of the stochastic variable $\xi$, and thus

$$
\left\langle\Psi_{i}, \Psi_{j}\right\rangle=\delta_{i j} c_{i}
$$

where $c_{i}$ is a constant depending on the polynomial basis. The relations between different polynomial bases is described by the Askey scheme; for further reading, see for instance [28]. Here it is sufficient to notice that the optimal basis in terms of convergence is Hermite polynomials if $\xi$ is distributed according to a Gaussian measure.

\subsection{Polynomial chaos expansion of Burgers' equation}

The polynomial chaos representation $u(x, t, \xi)=\sum_{i=0}^{\infty} u_{i} \Psi_{i}(\xi)$ is inserted into the Burgers' equation,

$$
u_{t}+u u_{x}=0,0 \leq x \leq 1
$$

which yields

$$
\sum_{i=0}^{\infty} \frac{\partial u_{i}}{\partial t} \Psi_{i}(\xi)+\left(\sum_{j=0}^{\infty} u_{j} \Psi_{j}(\xi)\right)\left(\sum_{i=0}^{\infty} \frac{\partial u_{i}}{\partial x} \Psi_{i}(\xi)\right)=0 .
$$

A stochastic Galerkin projection is performed by multiplying (8) by $\Psi_{k}(\xi)$ for non-negative integers $k$ and integrating over the probability domain $\Omega$. The orthogonality of the basis polynomials then yields a system of deterministic equations. By truncating the series (1) to a finite order $M$, the solution is projected onto a finite dimensional deterministic space. The result is a symmetric system of equations.

$$
\frac{\partial u_{k}}{\partial t}\left\langle\Psi_{k}^{2}\right\rangle+\sum_{i=0}^{M} \sum_{j=0}^{M} u_{i} \frac{\partial u_{j}}{\partial x}\left\langle\Psi_{i} \Psi_{j} \Psi_{k}\right\rangle=0 \quad \text { for } k=0,1, \ldots, M .
$$




\subsection{Modeling the solution with Hermite polynomials}

The probabilistic version of the Hermite polynomial basis functions is used since it is the most intuitive choice with regard to weight function and calculation of expected value and variance. The weight function of the inner products is the probability density function of the Gaussian distribution. This means that the inner product of two variables $u$ and $v$ coincides with the expected value of their product.

$$
\langle u, v\rangle=\int_{\Omega_{\mathrm{prob}}} u v f(\xi) d \xi=E(u v) .
$$

For probabilistic Hermite polynomials $H(\xi)$ of a Gaussian variable $\xi$ the double inner product defined in (6) is given by

$$
\left\langle H_{i} H_{j}\right\rangle=\delta_{i j} i !
$$

The triple inner product can be derived from a formula in [24]:

$\left\langle H_{i} H_{j} H_{k}\right\rangle=\left\{\begin{array}{ll}0 & \text { if } i+j+k \text { is odd or } \max (i, j, k)>s \\ \frac{i ! j ! k !}{(s-i) !(s-j) !(s-k) !} & \text { otherwise }\end{array}\right.$.

where $s=(i+j+k) / 2$.

\subsection{Derivation of an analytical solution}

By the Cameron-Martin theorem [3] the solution converges in the $L_{2}$ sense; in a probabilistic sense this implies mean square convergence, that is

$$
\lim _{M \rightarrow \infty} E\left[\left|u-\sum_{i=1}^{M} u_{i} \Psi_{i}\right|^{2}\right]=0 .
$$


In order to allow piecewise continuous solutions to the Burgers' equation we follow [9] and broaden the concept of solutions to the class of functions equivalent to $u$, denoted $C_{u}$, and define a normed space that does not require its elements to be smooth functions. We consider $u$ belonging to the space

$$
L_{w}^{2}(\Omega)=\left\{C_{u} \mid u \text { measurable, } \int_{\Omega_{\mathrm{prob}}} u^{2} f d \xi<\infty\right\}
$$

where the weight function $f$ is the probability density function of $\xi$.

Consider Burgers' equation (7) with stochastic boundary and initial conditions of the form

$$
\begin{aligned}
& u(x, 0, \xi)= \begin{cases}u_{L}=1+0.1 \xi & \text { if } x<x_{0} \\
u_{R}=-1+0.1 \xi & \text { if } x>x_{0}\end{cases} \\
& u(0, t, \xi)=u_{L}, u(1, t, \xi)=u_{R} \\
& \xi \in N(0,1),
\end{aligned}
$$

where we assume an initial shock location $x_{0} \in[0,1]$. In all experiments performed, $x_{0}=0.5$. For a fixed $\xi$ the initial discontinuity will travel with the shock speed $s=0.1 \xi$. For any given shock location $x_{s}$ at a time $t_{s}$, a unique $\xi_{s}$ given by

$$
\xi_{s}(x, t)=\frac{x_{s}-x_{0}}{0.1 t_{s}}
$$

exists. Using the relation (13), the analytical solution to the problem (7) and (12) is given by

$$
u(x, t, \xi)=\left\{\begin{array}{ll}
u_{L}=1+0.1 \xi & \text { if } \xi>\xi_{s} \\
u_{R}=-1+0.1 \xi & \text { if } \xi<\xi_{s}
\end{array} .\right.
$$


The boundary conditions are time-dependent but for short simulation times these can be assumed constant with negligible lack of accuracy. The solution clearly belongs to $L_{w}^{2}(\Omega)$ and thus

$$
\lim _{M \rightarrow \infty}\left\|u-\Pi_{f, M} u\right\|_{L_{w}^{2}(\Omega)}=0,
$$

where $\Pi_{f, M}$ is the projection operator to the space of Hermite polynomials of order $M$.

Since the analytical solution is known, the coefficients of the complete $\mathrm{PC}$ expansion $(M \rightarrow \infty)$ can be calculated for any given $i, x$ and $t$. We have $u_{i}(x, t)=\frac{1}{\left\langle\Psi_{i}^{2}\right\rangle} \int_{-\infty}^{\infty} u(x, t, \xi) \Psi_{i}(\xi) f(\xi) d \xi=\delta_{i 0}+0.1 \delta_{i 1}-\frac{2}{\left\langle\Psi_{i}^{2}\right\rangle} \int_{-\infty}^{\xi_{s}} \Psi_{i} f(\xi) d \xi$.

Using the recursion relation

$$
\Psi_{i}(\xi)=\xi \Psi_{i-1}(\xi)-\Psi_{i-1}^{\prime}(\xi)
$$

(14) can be written

$$
u_{i}(x, t)=\delta_{i 0}+0.1 \delta_{i 1}+\sqrt{\frac{2}{\pi}} \frac{\Psi_{i-1}\left(\xi_{s}\right) e^{-\xi_{s}^{2} / 2}}{i !},
$$

for $i \geq 1$. Differentiating (15) with respect to $x$ and $t$ results in

$$
\frac{\partial u_{i}}{\partial x}=\frac{\partial u_{i}}{\partial \xi_{s}} \frac{\partial \xi_{s}}{\partial x}=-\frac{2}{i !} \Psi_{i}\left(\xi_{s}(x, t)\right) f\left(\xi_{s}(x, t)\right) \frac{1}{0.1 t}
$$

and

$$
\frac{\partial u_{i}}{\partial t}=\frac{\partial u_{i}}{\partial \xi_{s}} \frac{\partial \xi_{s}}{\partial t}=\frac{2}{i !} \Psi_{i}\left(\xi_{s}(x, t)\right) f\left(\xi_{s}(x, t)\right) \frac{x-x_{0}}{0.1 t^{2}}
$$

from which it is clear that $u_{i}(x, t)$ is continuous in $x$ and $t$ for $x \in[0,1]$ and $t>$ 0 . (The same is true for $u_{0}$.) With an appropriate choice of initial function, 
the coefficients would be continuous also for $t=0$. For the treatment of a similar case of smooth coefficients of a discontinuous solution, see [7].

The solution to the truncated problem will be compared to the expected value and the variance of the analytical solution, given by

$$
E(u)=1-2 \int_{-\infty}^{\xi_{s}(x, t)} \frac{e^{-\xi^{2} / 2}}{\sqrt{2 \pi}} d \xi
$$

and

$$
\operatorname{Var}(u)=0.01+0.4 \frac{e^{-\xi_{s}^{2} / 2}}{\sqrt{2 \pi}}+4 \int_{-\infty}^{\xi_{s}} \frac{e^{-\xi^{2} / 2}}{\sqrt{2 \pi}} d \xi-4\left(\int_{-\infty}^{\xi_{s}} \frac{e^{-\xi^{2} / 2}}{\sqrt{2 \pi}} d \xi\right)^{2}
$$

These expressions can be generalized for different boundary conditions and polynomial bases.

\subsection{Truncated PC system for Burgers' equation}

The system of equations resulting from the stochastic Galerkin projection can be written in non-conservative matrix form as

$$
B u_{t}+A(u) u_{x}=0
$$

where $B$ is a positive definite constant diagonal matrix with the inner products of the basis polynomials and $A(u)$ is a symmetric matrix depending on $u$. As an illustration, the $3 \times 3$ system given by truncating the expansion (1) to $M=2$ with a Hermite polynomial basis for Burgers' equation is

$$
\left(\begin{array}{lll}
1 & 0 & 0 \\
0 & 1 & 0 \\
0 & 0 & 2
\end{array}\right)\left(\begin{array}{l}
u_{0} \\
u_{1} \\
u_{2}
\end{array}\right)_{t}+\left(\begin{array}{ccc}
u_{0} & u_{1} & 2 u_{2} \\
u_{1} & u_{0}+2 u_{2} & 2 u_{1} \\
2 u_{2} & 2 u_{1} & 2 u_{0}+8 u_{2}
\end{array}\right)\left(\begin{array}{l}
u_{0} \\
u_{1} \\
u_{2}
\end{array}\right)_{x}=0
$$


or the equivalent non-symmetric form used in the simulations

$$
\left(\begin{array}{l}
u_{0} \\
u_{1} \\
u_{2}
\end{array}\right)_{t}+\left(\begin{array}{ccc}
u_{0} & u_{1} & 2 u_{2} \\
u_{1} & u_{0}+2 u_{2} & 2 u_{1} \\
u_{2} & u_{1} & u_{0}+4 u_{2}
\end{array}\right)\left(\begin{array}{c}
u_{0} \\
u_{1} \\
u_{2}
\end{array}\right)_{x}=0 .
$$

From the system of equations it is clear that if no uncertainty is introduced in the polynomial chaos expansion of the initial and boundary conditions, all coefficients $u_{i}=0, \forall i \geq 1$ and the system is reduced to the scalar, deterministic Burgers' equation. The deterministic Burgers' equation is thus a special case of the stochastic Burgers' equation.

\subsection{Conservation form}

Burgers' equation (7) should be expressed in conservation form to obtain correct shock speed, i.e.

$$
u_{t}+\frac{\partial}{\partial x} f(u)=0
$$

where $f(u)=u^{2} / 2$. Both the conservative and the non-conservative formulations will be studied in the following sections.

Using the conservative form (21), a stochastic Galerkin projection on the polynomial chaos expansion truncated to $M$ terms gives

$$
\left\langle\Psi_{k}^{2}\right\rangle \frac{\partial u_{k}}{\partial t}+\frac{\partial}{\partial x} \frac{1}{2} \sum_{i=0}^{M} \sum_{j=0}^{M} u_{i} u_{j}\left\langle\Psi_{i} \Psi_{j} \Psi_{k}\right\rangle=0,
$$

for $k=0,1, \ldots, M$. In matrix form with the matrices $A$ and $B$ defined as before, (22) can be written

$$
B u_{t}+\frac{\partial}{\partial x} f(u)=0, f(u)=\frac{1}{2} A(u) u .
$$

As a comparison, the system $u_{t}+u u_{x}=0$ lead to (18). Note that the matrix $A=A(u)$ occurs in both the conservative and non-conservative form. 


\subsection{Diagonalization}

For various purposes, such as analysis of well-posedness, design of dissipation operators and analysis of characteristics, the system (18) is diagonalized. $A$ and $B$ are both positive definite and symmetric matrices and it can be shown that $B^{-1} A$ has real valued eigenvalues and eigenvectors. See [11] for further details and proof.

Assuming $A$ constant, let $\Lambda$ denote a diagonal matrix with the eigenvalues $\lambda_{i}$ of $B^{-1} A$ on the main diagonal and $V$ a matrix where the columns are the linearly independent eigenvectors. Eq. (18) is rewritten as

$$
w_{t}+\Lambda w_{x}=0
$$

where $w=V^{-1} u$.

Assuming non-zero eigenvalues, $\Lambda$ can be split according to the sign of its eigenvalues as $\Lambda=\Lambda^{+}+\Lambda^{-}$. Introducing the split scheme into the system of equations gives

$$
w_{t}+\Lambda^{+} w_{x}+\Lambda^{-} w_{x}=0
$$

This form will be used in the following sections.

\section{Well-posedness}

A problem is well posed if the solution exists, is unique and depends continuously on the problem data. An initial-boundary-value problem given by

$$
\begin{array}{ll}
u_{t}+\mathcal{H}\left(x, t, \frac{\partial}{\partial x_{i}}\right) u=F(x, t) & x \in \Omega_{\text {phys }} \quad t \geq 0 \\
u=f(x) & x \in \Omega_{\text {phys }} \quad t=0 \\
L u=g(t) & x \in \Gamma_{\text {phys }} \quad t \geq 0
\end{array}
$$


is strongly well posed if the solution exists, is unique and is subject to the estimate

$$
\|u\|_{\Omega_{\mathrm{phys}}}^{2}+\int_{0}^{t}\|u\|_{\Gamma_{\mathrm{phys}}}^{2} d \tau \leq K_{c} e^{\eta_{c} t}\left(\|f\|_{\Omega_{\mathrm{phys}}}^{2}+\int_{0}^{t}\|F\|_{\Omega_{\mathrm{phys}}}^{2}+\|g\|_{\Gamma_{\mathrm{phys}}}^{2} d \tau\right) .
$$

$K_{c}$ and $\eta_{c}$ are independent of $F, f$ and $g$. See $[13,17]$ for more details.

The solution of (26) requires initial and boundary data. The data depend on the expected conditions and the distribution of the uncertainty introduced; the stochastic Galerkin procedure is again used to determine the polynomial chaos coefficients for the initial and boundary values. In this section we will show that the truncated system resulting from a truncated PC expansion is well-posed if correct boundary conditions are given.

In the rest of this section, we assume $u$ to be smooth. Consider the continuous problem in split form [22]:

$$
B u_{t}+\beta \frac{\partial}{\partial x}\left(\frac{A}{2} u\right)+(1-\beta) A u_{x}=0,0 \leq x \leq 1 .
$$

Multiplication by $u^{T}$ and integration over the spatial domain $\Omega_{\text {phys }}=[0,1]$ yields

$$
\int_{0}^{1} u^{T} B u_{t} d x+\beta \int_{0}^{1} u^{T} \frac{\partial}{\partial x}\left(\frac{A}{2} u\right) d x+(1-\beta) \int_{0}^{1} u^{T} A u_{x} d x=0 .
$$

Integration by parts and the observation that $B$ is positive definite gives

$$
\frac{1}{2} \frac{\partial}{\partial t}\|u\|_{B}^{2}=-\frac{\beta}{2}\left[u^{T} A u\right]_{x=0}^{x=1}+\frac{\beta}{2} \int_{0}^{1} u_{x}^{T} A u d x-(1-\beta) \int_{0}^{1} u^{T} A u_{x} d x .
$$

We choose $\beta$ such that

$$
\frac{\beta}{2}-(1-\beta)=0 \Leftrightarrow \beta=\frac{2}{3},
$$


which is inserted into (28), yielding

$$
\frac{\partial}{\partial t}\|u\|_{B}^{2}=-\frac{2}{3}\left[u^{T} A u\right]_{x=0}^{x=1}=\frac{2}{3}\left(w_{0}^{T}\left(\Lambda_{0}^{+}+\Lambda_{0}^{-}\right) w_{0}-w_{1}^{T}\left(\Lambda_{1}^{+}+\Lambda_{1}^{-}\right) w_{1}\right) .
$$

where $A(u)$ has been diagonalized at the boundaries according to Sec. 2.6. Boundary conditions are imposed on the resulting incoming characteristic variables which correspond to $\Lambda^{+}$for $x=0$ and $\Lambda^{-}$for $x=1$. On the left boundary, the conditions are set such that

$$
\left(w_{0}\right)_{i}=\left(V^{-1} u(x=0)\right)_{i}=\left(g_{0}\right)_{i} \text { if } \lambda_{i}>0
$$

and on the right boundary

$$
\left(w_{1}\right)_{i}=\left(V^{-1} u(x=1)\right)_{i}=\left(g_{1}\right)_{i} \text { if } \lambda_{i}<0 .
$$

The boundary norm is defined as

$$
\|w\|_{\Gamma_{\text {phys }}}^{2}=w^{T} \Lambda^{+} w-w^{T} \Lambda^{-} w=w^{T}\left(\Lambda^{+}+\left|\Lambda^{-}\right|\right) w=w^{T}|\Lambda| w \text { for } x=0,1 .
$$

Inserting the boundary conditions and integrating Eq. (29) over time gives

$\|u\|_{\Omega_{\text {phys }}}^{2}+\frac{2}{3} \int_{0}^{t}\left\|w_{0}\right\|_{\Gamma_{\text {phys }}}^{2}+\left\|w_{1}\right\|_{\Gamma_{\text {phys }}}^{2} d \tau \leq\|f\|_{\Omega_{\text {phys }}}^{2}+\frac{4}{3} \int_{0}^{t}\left\|g_{0}\right\|_{\Gamma_{\text {phys }}}^{2}+\left\|g_{1}\right\|_{\Gamma_{\text {phys }}}^{2} d \tau$.

Since $\|w\| \leq\left\|V^{-1}\right\|\|u\| \leq C\|u\|$ for some $C<\infty$, the estimate (30) is in the form of Eq. (27).

Uniqueness follows directly from (30). Assume $u$ and $v$ are two different solutions to the Burgers' equation with given conditions. Then $u-v$ is a solution to the homogeneous system. The energy estimate (30) with zero data shows that this solution equals zero everywhere and therefore $u=v$ and the solution is unique. Since existence is trivial in this case (a hyperbolic problem with correct number of boundary conditions), we have shown well-posedness. 
Remark: The assumption that $u$ is smooth is actually true for an infinite number of terms of the polynomial chaos expansion and $t>0$.

\section{Energy estimates for stability analysis}

Although the problems of interests are stochastic, the problem that arises from the stochastic Galerkin projection is strictly deterministic. For such a problem, well-known numerical techniques can be used to ensure stable and accurate solutions.

\subsection{Summation by parts operators}

Summation by parts (SBP) is the discrete equivalent to integration by parts. A difference operator $P^{-1} Q$ has the SBP property if it has the characteristic form

$$
Q+Q^{T}=\left(\begin{array}{ccccc}
-1 & & & & 0 \\
& 0 & & & \\
& & \ddots & & \\
& & & 0 & \\
0 & & & & 1
\end{array}\right) \text { and } P=P^{T}>0 .
$$

$P$ is the so-called diagonal norm. SBP operators are used for approximations of spatial derivatives. Their usefulness lies in the possibility of expressing energy decay in terms of known boundary values, exactly as in the continuous case $[23,16]$.

\subsection{Stability of the semi-discretized problem}

Consider the continuous problem in non-conservative form

$$
B u_{t}+A u_{x}=0 .
$$


We will use the so-called penalty technique [16] to impose boundary conditions for the discrete problem.

In the analysis below, let $E_{0}=\left(e_{i j}\right)$ where $e_{11}=1, e_{i j}=0, \forall i, j \neq 1$ and $E_{n}=\left(e_{i j}\right)$ where $e_{n n}=1, e_{i j}=0, i, j \neq n$. Define the block diagonal matrix $A_{g}$ where the diagonal blocks are the symmetric matrices $A(u(x))$. With penalty matrices $\Sigma_{0}$ and $\Sigma_{1}$ corresponding to the left and right boundaries respectively, the discretized system can be expressed as

$$
(I \otimes B) u_{t}+A_{g}\left(P^{-1} Q \otimes I\right) u=\left(P^{-1} \otimes I\right)\left(E_{0} \otimes \Sigma_{0}\right)\left(u-g_{0}\right)+\left(P^{-1} \otimes I\right)\left(E_{n} \otimes \Sigma_{1}\right)\left(u-g_{1}\right) .
$$

Similarly, the conservative system,

$$
B u_{t}+\frac{1}{2} \frac{\partial}{\partial x}(A u)=0
$$

can be discretized as

$$
(I \otimes B) u_{t}+\frac{1}{2}\left(P^{-1} Q \otimes I\right) A_{g} u=\left(P^{-1} \otimes I\right)\left(E_{0} \otimes \Sigma_{0}\right)\left(u-g_{0}\right)+\left(P^{-1} \otimes I\right)\left(E_{n} \otimes \Sigma_{1}\right)\left(u-g_{1}\right) .
$$

Neither of the formulations (32) nor (33) will lead to an energy estimate. However, the non-conservative and conservative forms can be combined to get an energy estimate by using the summation by parts property.

A linear combination of the conservative and the non-conservative form is used for the energy estimates, just as in the continuous case, see section 3. The split form is given by

$$
\begin{aligned}
(I \otimes B) u_{t}+\beta & \frac{1}{2}\left(P^{-1} Q \otimes I\right) A_{g} u+(1-\beta) A_{g}\left(P^{-1} Q \otimes I\right) u= \\
& =\left(P^{-1} \otimes I\right)\left[\left(E_{0} \otimes \Sigma_{0}\right)\left(u-g_{0}\right)+\left(E_{n} \otimes \Sigma_{1}\right)\left(u-g_{1}\right)\right] .
\end{aligned}
$$


With multiplication by $u^{T}(P \otimes I)$ the system can be written as

$$
\begin{array}{r}
u^{T}(P \otimes B) u_{t}+\frac{\beta}{2} u^{T}(Q \otimes I) A_{g} u+(1-\beta) u^{T}(P \otimes I) A_{g}\left(P^{-1} Q \otimes I\right) u= \\
=u^{T}\left(E_{0} \otimes \Sigma_{0}\right)\left(u-g_{0}\right)+u^{T}\left(E_{n} \otimes \Sigma_{1}\right)\left(u-g_{1}\right) .
\end{array}
$$

We will use the commutativity property

$$
A_{g}=(P \otimes I) A_{g}\left(P^{-1} \otimes I\right)
$$

We add the transpose to (35) and use (36) to get

$$
\begin{aligned}
\frac{\partial}{\partial t}\|u\|_{(P \otimes B)}^{2}+ & \frac{\beta}{2} u^{T}\left((Q \otimes I) A_{g}+A_{g}\left(Q^{T} \otimes I\right)\right) u+ \\
& +(1-\beta) u^{T}\left(A_{g}(Q \otimes I)+\left(Q^{T} \otimes I\right) A_{g}\right) u= \\
& =2 u^{T}\left(E_{0} \otimes \Sigma_{0}\right)\left(u-g_{0}\right)+2 u^{T}\left(E_{n} \otimes \Sigma_{1}\right)\left(u-g_{1}\right) .
\end{aligned}
$$

As before, we choose $\beta$ such that

$$
\frac{\beta}{2}=1-\beta \Leftrightarrow \beta=\frac{2}{3}
$$

By the summation by parts property (31) this yields the desired form

$$
\begin{aligned}
\frac{\partial}{\partial t}\|u\|_{(P \otimes B)}^{2}=\frac{2}{3}\left(u_{x=0}^{T} A u_{x=0}-u_{x=1}^{T} A u_{x=1}\right)+ & 2 u_{x=0}^{T} \Sigma_{0}\left(u_{x=0}-g_{0}\right)+ \\
& +2 u_{x=1}^{T} \Sigma_{1}\left(u_{x=1}-g_{1}\right) .
\end{aligned}
$$

Restructuring (38) yields

$$
\begin{aligned}
\frac{\partial}{\partial t}\|u\|_{(P \otimes B)}^{2}=u_{x=0}^{T}\left(\frac{2}{3} A+2 \Sigma_{0}\right) u_{x=0}-2 u_{x=0}^{T} \Sigma_{0} g_{0}-u_{x=1}^{T}\left(\frac{2}{3} A\right. \\
\left.-2 \Sigma_{1}\right) u_{x=1}-2 u_{x=1}^{T} \Sigma_{1} g_{1} .
\end{aligned}
$$


Stability is achieved by a proper choice of the penalty matrices $\Sigma_{0}$ and $\Sigma_{1}$. For that purpose $A$ is split according to the sign of its eigenvalues as

$$
A=A^{+}+A^{-} \text {where } A^{+}=x^{T} \Lambda^{+} x \text { and } A^{-}=x^{T} \Lambda^{-} x .
$$

Choose $\Sigma_{0}$ and $\Sigma_{1}$ such that $\frac{2}{3} A^{+}+2 \Sigma_{0}=-\frac{2}{3} A^{+} \Leftrightarrow \Sigma_{0}=-\frac{2}{3} A^{+}$and $\frac{2}{3} A^{-}-2 \Sigma_{1}=\frac{2}{3} A^{-} \Leftrightarrow \Sigma_{1}=\frac{2}{3} A^{-}$. We now get the energy estimate

$$
\begin{gathered}
\frac{\partial}{\partial t}\|u\|_{(P \otimes B)}^{2}=-\frac{2}{3}\left(u_{x=0}-g_{0}\right)^{T} A^{+}\left(u_{x=0}-g_{0}\right)+\frac{2}{3}\left[u_{x=0}^{T} A^{-} u_{x=0}+g_{0}^{T} A^{+} g_{0}\right] \\
-\frac{2}{3}\left[u_{(x=1)}^{T} A^{+} u_{(x=1)}+g_{1}^{T} A^{-} g_{1}\right]+\frac{2}{3}\left(u_{(x=1)}-g_{1}\right)^{T} A^{-}\left(u_{(x=1)}-g_{1}\right), \quad(41)
\end{gathered}
$$

which shows that the system is stable.

Remark: in the numerical calculations we use (33) for correct shock speed, see [15].

\section{Artificial dissipation operators}

An artificial dissipation operator is a discretized even order derivative which is added to the system to allow stable and accurate solutions to be obtained in the presence of solution discontinuities. The artificial dissipation is designed to transform the global discretization into a one-sided operator close to the shock location. Depending on the accuracy of the difference scheme, this require one or more dissipation operators. The accuracy of the difference approximation is chosen as high as possible within the computational stencil of the difference approximation of the system matrix. All dissipation operators used here are of the form

$$
A_{2 k}=-\Delta x P^{-1} \tilde{D}_{k}^{T} B_{w} \tilde{D}_{k},
$$


where $P^{-1}$ is the diagonal norm of the first derivative as before, $\tilde{D}$ is an approximation of $(\Delta x)^{k} \partial^{k} / \partial x^{k}$ and $B_{w}$ is a diagonal positive definite matrix. In most cases here, $B_{w}$ is replaced by a single constant $\beta_{w}$. An appropriate choice of dissipation constant results in an upwind scheme, suitable for problems where shocks evolve. For further reading about the design of artificial dissipation operators we refer to [16].

The complete difference approximation (33) augmented with artificial dissipation is given by

$$
\begin{array}{r}
(P \otimes B) u_{t}+\frac{1}{2}(Q \otimes I) A_{g} u-\left(E_{0} \otimes \Sigma_{0}\right)\left(u-g_{1}\right)-\left(E_{n} \otimes \Sigma_{1}\right)\left(u-g_{1}\right)= \\
=-\Delta x \sum_{k}\left(\tilde{D}_{k}^{T} \otimes B\right) B_{w, k}\left(\tilde{D}_{k} \otimes I\right) u,
\end{array}
$$

where $B_{w, k}$ is a possibly non-constant weight matrix to be determined and $k=1,2$ for the fourth order accurate SBP operator.

Determining $B_{w}$ in (42) requires estimates of the eigenvalues $\lambda_{j}$ of $B^{-1} A$ for $j=0, \ldots, M$; the largest eigenvalue is typically sufficient. For the system of equations generated by polynomial chaos expansion of Burgers' equation, $\max |\lambda|$ is not always known. Since the only non-zero polynomial coefficients on the boundaries are $u_{0}$ and $u_{1}$ and since the polynomial chaos expansion converges in the $L_{2}$ sense, a reasonable approximation of the maximum eigenvalue of $B^{-1} A$ is

$$
|\lambda|_{\max } \approx\left|u_{0}\right|+M\left|u_{1}\right|
$$

where $M$ is the order of polynomial chaos expansion. This estimate is justified by the eigenvalue analysis performed in the next section as well as by computational results. For the dissipation operators $A_{2}$ and $A_{4}$ in the 
simulations, we use

$$
B_{w, 2}=\operatorname{diag}\left(\frac{\left(\left|u_{0}\right|+M\left|u_{1}\right|\right)}{6 \Delta x}\right), B_{w, 4}=\operatorname{diag}\left(\frac{\left(\left|u_{0}\right|+M\left|u_{1}\right|\right)}{24 \Delta x}\right) .
$$

The second order dissipation operator is only applied close to discontinuities.

\section{Time integration}

The increase in simulation cost associated with higher order systems is due to a number of factors. The size of the system depends both on the number of terms in the truncated polynomial chaos expansion and the spatial mesh size.

For the Kronecker product $A \otimes B$ the relation

$$
\lambda_{A \otimes B}^{i, j}=\lambda_{A}^{i} \lambda_{B}^{j}
$$

holds, where the indices $i, j$ denotes all the eigenvalues of $A$ and $B$ respectively. This enables a separate analysis of the eigenvalues corresponding to the polynomial chaos expansion and the eigenvalues of the total spatial difference operator $D$. Assuming constant coefficients, the maximum system eigenvalue is limited by

$$
\lambda_{\max } \leq\left(\max \lambda_{D}\right)\left(\max \lambda_{B^{-1} A}\right)
$$

The estimate (47) in combination with (44) will be used in order to obtain estimates of the time step constraint.

\subsection{Eigenvalue approximation}

Analytic eigenvalues for the matrix $B^{-1} A$ can only be obtained for a small number of polynomial chaos coefficients and therefore approximations 
are needed. Even though most eigenvalues of interest in this report can be calculated exactly for every particular case, a general estimate is of interest. The approximation of the largest eigenvalue of the scaled system matrix $B^{-1} A$ is calculated from solution values on the boundaries, which are the only values known a priori. For smooth solutions with boundary conditions where the polynomial chaos coefficients $u_{i}$ are equal to 0 for $i>1$, the higher order coefficients tend to remain small compared to lower order coefficients (strong probabilistic convergence). For solutions where a shock is developing, higher order polynomial chaos coefficients might grow and the approximation of the largest eigenvalue based on boundary values is likely to be a less accurate estimate.

To get estimates of the eigenvalues, the system of equations can be written

$$
u_{t}+B^{-1}\left(\sum_{i=0}^{M} A_{i}\left(u_{i}\right)\right) u_{x}=0,
$$

where $A(u)=A_{i} u_{i}$ is a linear combination of the polynomial chaos coefficients. The eigenvalue approximation used here is given by

$$
\begin{aligned}
\max \lambda_{B^{-1} A}=\max \frac{x^{T}\left(B^{-1} \sum A_{i} u_{i}\right) x}{x^{T} x} \leq \sum_{i} \max & \frac{x_{i}^{T} B^{-1} A_{i} x_{i}}{x_{i}^{T} x_{i}}\left|u_{i}\right|= \\
& =\sum_{i}\left|u_{i}\right| \max \left|\lambda_{i}\right| .
\end{aligned}
$$

Since $B^{-1} A_{0}\left(u_{0}\right)=u_{0} I$, this approximation coincides with the exact eigenvalue for boundary value with $u_{i}=0$ for $i>1$. This can be seen by observing that if $x_{1}$ is an eigenvector with corresponding eigenvalue $\lambda$ for the matrix $B^{-1} A_{1}$ then $B^{-1} A_{1} x=\lambda x$ and

$$
B^{-1}\left(A_{1} u_{1}+A_{0} u_{0}\right) x=u_{1} \lambda x+u_{0} I x=\left(u_{1} \lambda+u_{0}\right) x
$$


so $u_{1} \lambda+u_{0}$ and $x_{1}$ are eigenvalue and eigenvector to the matrix $B^{-1}\left(A_{0} u_{0}+\right.$ $\left.A_{1} u_{1}\right)=B^{-1} A$. This shows that (44) is an appropriate eigenvalue approximation for problems where only $u_{0}$ and $u_{1}$ are non-zero on the boundaries.

For a given boundary condition, the maximum eigenvalue of $A_{0}$ corresponding to the deterministic part of the condition does not change with increasing number of polynomial chaos coefficients. However, the largest eigenvalue contribution from $A_{1}$ grows with the number of polynomial chaos coefficients.

The eigenvalue approximation (44) is in general of the same order of magnitude as the largest eigenvalue in the interior of the domain but might have to be adjusted to remove all oscillations. The exact value is problem specific and an estimate based on the interior values requires knowledge about the solution of the problem.

\subsection{Efficiency of the polynomial chaos method}

The convergence of the polynomial chaos expansion is investigated by measuring the discrete Euclidean error norm of the variance and the expected value. For a discretization with $m$ spatial grid points, we have

$$
\left\|\epsilon_{E x p}\right\|^{2}=\frac{1}{m-1} \sum_{i=1}^{m}\left(\mathrm{E}[u]_{m}-\mathrm{E}\left[u_{r e f}\right]_{m}\right)^{2}
$$

and

$$
\left\|\epsilon_{\operatorname{Var}}\right\|^{2}=\frac{1}{m-1} \sum_{i=1}^{m}\left(\operatorname{Var}[u]_{m}-\operatorname{Var}\left[u_{r e f}\right]_{m}\right)^{2}
$$

where $u_{r e f}$ denotes the analytical solution. Consider the model problem (12); the problem is solved with the Monte Carlo method and PC until time $t=0.3$. Accuracy (measured as the norm of the difference between the actual 


\begin{tabular}{|l|lllll|}
\hline $\mathrm{N}$ & 10 & 50 & 100 & 400 & 1600 \\
\hline$\left\|\epsilon_{E x p}\right\|$ & 0.122 & 0.0374 & 0.0344 & 0.0257 & 0.0151 \\
$\left\|\epsilon_{\text {Var }}\right\|$ & 0.127 & 0.0589 & 0.0426 & 0.0283 & 0.0189 \\
$\mathrm{~T}(\mathrm{~s})$ & 240 & 1180 & 2390 & 9350 & 38460 \\
\hline
\end{tabular}

Table 1: Convergence to (16) and (17) with the Monte Carlo method, $m=400, t=0.3$.

\begin{tabular}{|l|llll|}
\hline $\mathrm{M}$ & 2 & 4 & 6 & 8 \\
\hline$\left\|\epsilon_{\text {Exp }}\right\|$ & 0.113 & 0.0544 & 0.0164 & 0.0150 \\
$\left\|\epsilon_{\text {Var }}\right\|$ & 0.147 & 0.122 & 0.0409 & 0.0630 \\
$T(\mathrm{~s})$ & 126 & 636 & 4180 & 10900 \\
\hline
\end{tabular}

Table 2: Convergence to (16) and (17) with the polynomial chaos method, $m=400$, $t=0.3$.

solution and the analytical solution) and simulation cost are shown in Table 1 for the Monte Carlo method and Table 2 for the PC expansions.

For this highly non-linear and discontinuous problem, the polynomial chaos method is more efficient than the Monte Carlo method with low accuracy requirements. The convergence properties of these solutions are affected by the spatial grid size and the accuracy of imposed artificial dissipation and no general conclusion of the relative performances of the two methods will be drawn here. As will be further illustrated in the section on analysis of characteristics, the solution coefficients of the truncated system are discontinuous approximations to the analytical coefficients which are smooth. Even though the PC results do converge for this problem, the low order expansions are qualitatively very different from the analytical solution, see for instance Figure 1. Also, note that excessive use of artificial dissipation is likely to 

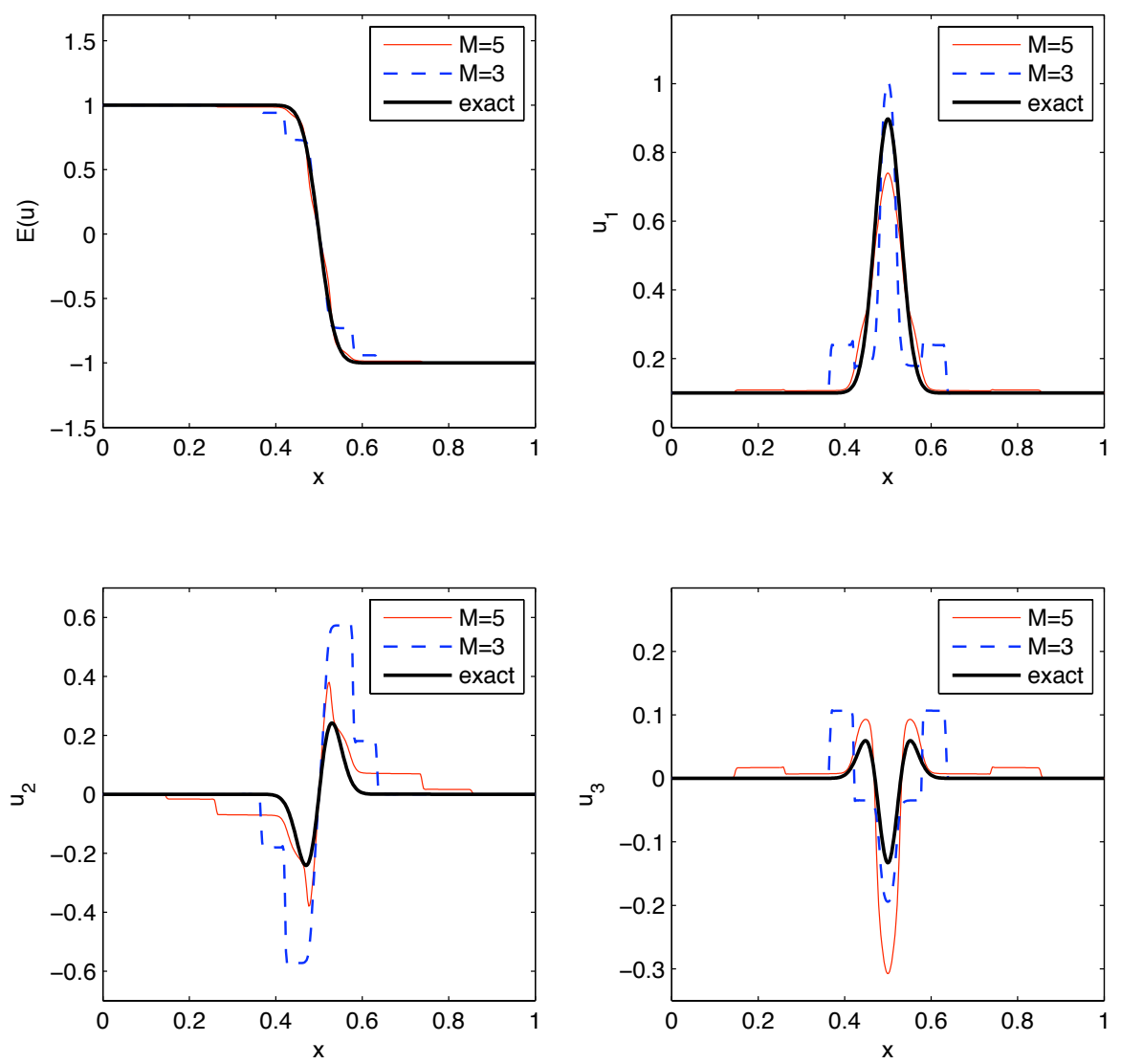

Figure 1: The first four PC coefficients, $t=0.3, M=5$ and $M=3, m=400$. 
produce a solution closer to the analytical solution for lower order expansions. Note that, as expected spatial grid refinement leads to convergence to the true solution of the truncated system but does not get any closer to the analytical solution.

The use of artificial dissipation proportional to the largest eigenvalue makes the solutions of large order expansions dissipative and spatial grid refinement is needed for an accurate solution. This can be seen in Table 2, where the accuracy of the variance decreases with large $M$.

\subsection{Numerical convergence}

The convergence of the computed polynomial chaos coefficients, the expected value and the variance of the truncated system is investigated and comparisons to the analytical solution derived in section 2.3 are presented.

As mentioned earlier, the numerical results obtained for a small number of expansion terms is expected to be a poor approximation to the analytical solution; this is confirmed by the mesh refinement study reported in Figure 1 for $M=5$. In this particular application, the analytical solution admits continuous (smooth) coefficients in spite of the discontinuous initial condition; on the other hand, the coefficients of the truncated system are discontinuous.

Interestingly, the difference between the computed coefficients corresponding to a finite PC expansion $\left(u_{i}\right.$ for $\left.i \leq M\right)$ and the analytical $(M=\infty)$ coefficients indicates that a poorly resolved numerical solution with excessive

dissipation might be qualitatively closer to the analytical solution than a grid converged solution to the truncated system. Figure 2 and Table 3 illustrates this phenomenon of illusory convergence. 


\begin{tabular}{|l|ccc|}
\hline $\mathrm{M}$ & 3 & 3 (dissipative) & 4 \\
\hline$\left\|\epsilon_{\text {Exp }}\right\|$ & 0.0354 & 0.0173 & 0.0374 \\
$\left\|\epsilon_{\text {Var }}\right\|$ & 0.0918 & 0.0370 & 0.0723 \\
\hline
\end{tabular}

Table 3: Norms of errors for dissipative and non-dissipative solutions.
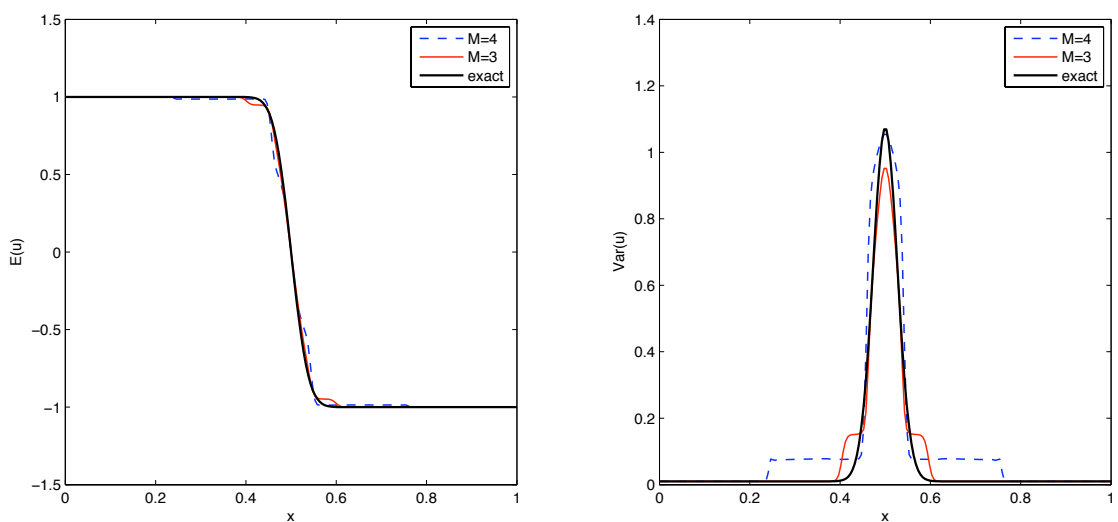

Figure 2: Dissipative solution on course grid $(m=200)$, computed for $M=3$ and nondissipateive solution for $M=4$.

The discrepancy between the truncated solution for $M=3$ and the analytical solution is also illustrated in Figure 3. The coefficients do not converge to the analytical solution when the spatial grid is refined (Figure 3a, left). Instead the coefficients converge numerically to a reference solution corresponding to a numerical solution obtained with a large number of gridpoints (Figure 3a, right). For the 7th order expansion, the solution is sufficiently close the the solution of the analytical problem to exhibit spatial numerical convergence of the first four coefficients to the analytical coefficients (Figure $3 \mathrm{~b})$. 

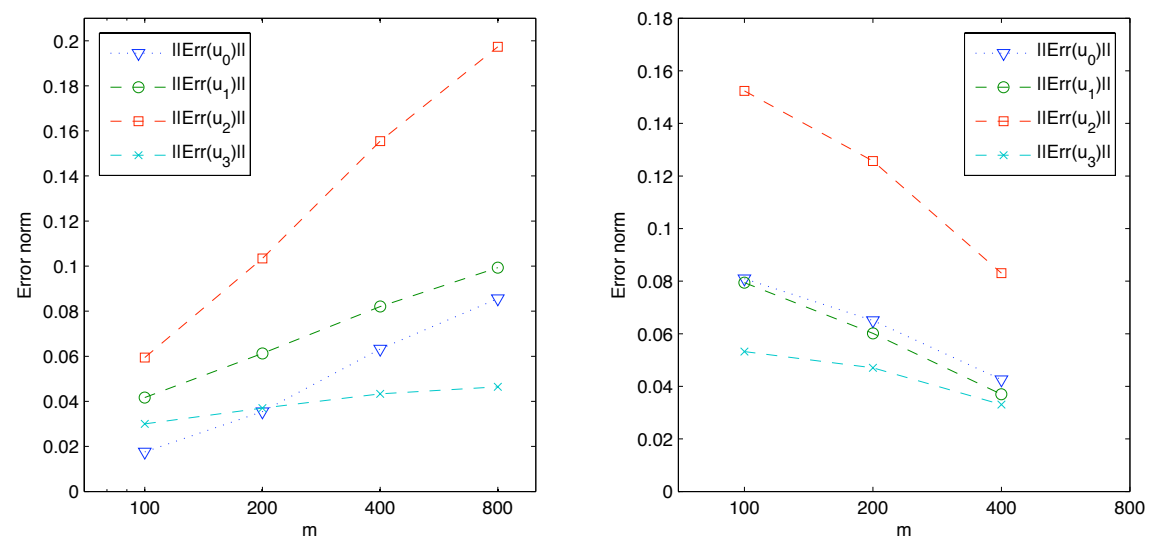

(a) $M=3$. Norm of the error relative to the analytical solution (left) and error relative to the finest grid solution, $m=800$ (right).
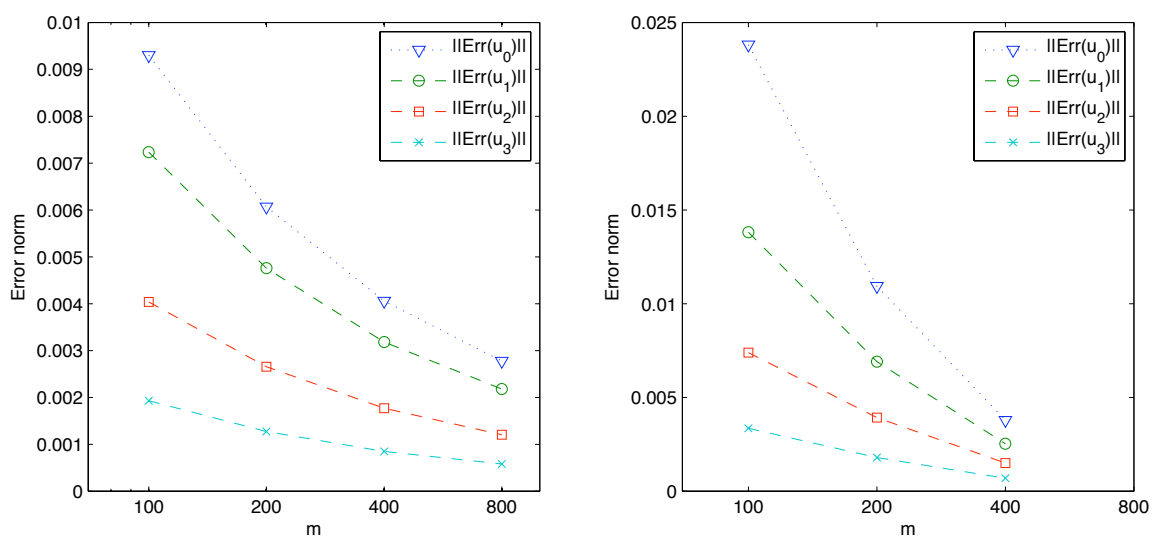

(b) $M=7$. Norm of the error relative to the analytical solution (left) and error relative to the finest grid solution, $m=800$ (right).

Figure 3: Convergence of the first chaos coefficients. Note the different scales in the figures.

The variance calculated for $M=7$ appears to converge to a function that is close but not equal to the analytical variance given by (17), see Figure 4 . 

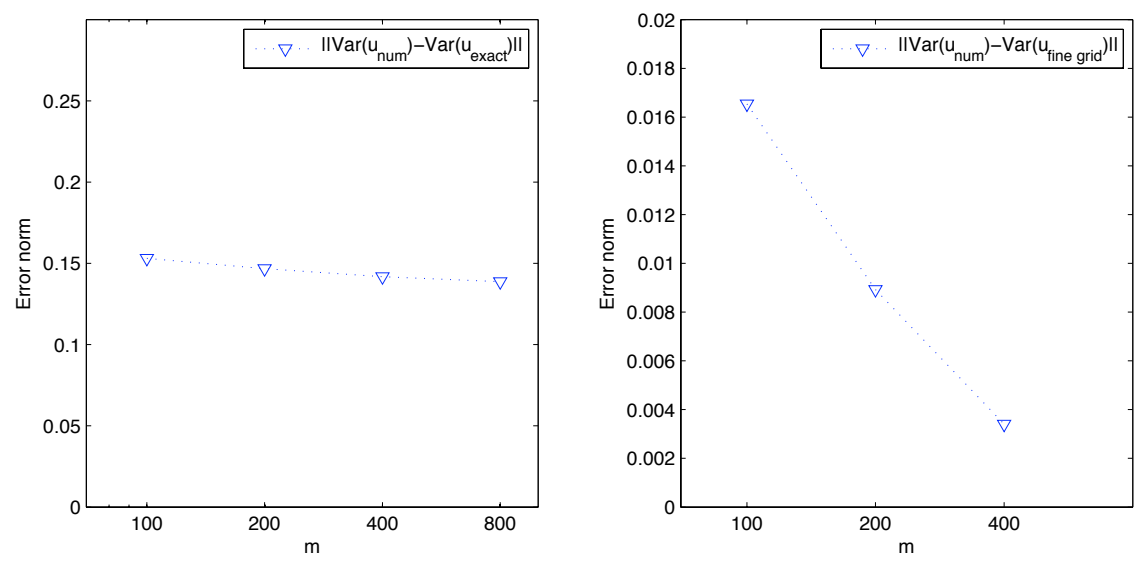

Figure 4: $M=7$. Convergence of the variance. Norm of the error relative to the analytical variance (left) and error relative to the finest grid variance, $m=800$ (right).

\section{Theoretical results and interpretation}

\subsection{Analysis of characteristics: disturbed cosine wave}

In this section, the characteristics of the stochastic Burgers' equation with $M=1$ (truncated to $2 \times 2$ system) will be investigated to give a qualitative measure of the time development of the solution. The system is given by

$$
\left(\begin{array}{l}
u_{0} \\
u_{1}
\end{array}\right)_{t}+\left(\begin{array}{ll}
u_{0} & u_{1} \\
u_{1} & u_{0}
\end{array}\right)\left(\begin{array}{l}
u_{0} \\
u_{1}
\end{array}\right)_{x}=0 .
$$

With $w_{1}=u_{0}+u_{1}$ and $w_{2}=u_{0}-u_{1}$, (51) can be diagonalized and rewritten

$$
\left(\begin{array}{l}
w_{1} \\
w_{2}
\end{array}\right)_{t}+\left(\begin{array}{cc}
w_{1} & 0 \\
0 & w_{2}
\end{array}\right)\left(\begin{array}{l}
w_{1} \\
w_{2}
\end{array}\right)_{x}=0 .
$$

Equation (52) is the original Burgers' equation for $w_{1}, w_{2}$ and the shock speeds are given by

$$
s_{w_{1}}=\frac{\left[f\left(w_{1}\right)\right]}{\left[w_{1}\right]}=\frac{w_{1 R}+w_{1 L}}{2}=\overline{u_{0}}+\overline{u_{1}}
$$


and

$$
s_{w_{2}}=\frac{\left[f\left(w_{2}\right)\right]}{\left[w_{2}\right]}=\frac{w_{2 R}+w_{2 L}}{2}=\overline{u_{0}}-\overline{u_{1}}
$$

where we have introduced the mean over the shock, $\bar{u}_{i}=\left(u_{i L}+u_{i R}\right) / 2$. Double brackets [ ] denotes the jump in a quantity over a discontinuity. Similarly to (53) and (54), with the non-diagonalized system in conservation form, the propagation speeds of discontinuities in $u_{0}, u_{1}$ are given by

$$
s_{u_{0}}=\frac{\left[\left(u_{0}^{2}+u_{1}^{2}\right) / 2\right]}{\left[u_{0}\right]}=\overline{u_{0}}+\overline{u_{1}} \frac{\left[u_{1}\right]}{\left[u_{0}\right]}
$$

and

$$
s_{u_{1}}=\frac{\left[u_{0} u_{1}\right]}{\left[u_{1}\right]}=\overline{u_{0}}+\overline{u_{1}} \frac{\left[u_{0}\right]}{\left[u_{1}\right]} .
$$

The analysis of characteristics $w_{1}$ and $w_{2}$ describes the behavior and emergence of discontinuities in the coefficients $u_{0}$ and $u_{1}$ of the truncated system. However, the coefficients of the solution to the problem given by the infinite $\mathrm{PC}$ expansion are smooth (except for $t=0$ for the Riemann problem). Diagonalization of large systems is not feasible but we can obtain expressions for the shock speeds of the coefficients. For instance, the expression (55) for the shock speed in $u_{0}$ can be generalized for expansions of order $M$ as

$$
s_{u_{0}}=\sum_{i=0}^{M} \overline{u_{i}} \frac{\left[u_{i}\right]}{\left[u_{0}\right]} i !
$$

In the assumption that only one Gaussian variable $\xi$ is introduced, and the uncertainty is (linearly) proportional to $\xi$ only a limited number of different values of the correlation coefficient between the left and right state can occur. Since we are also assuming the same model for the left and right state uncertainties, only a few combinations of covariance matrices describing their correlation are realizable. With the assumptions made here, the dependence 
between the two states is determined by the correlation coefficient $\rho_{L R}$, which for these cases is either 1 or -1 .

\section{$\operatorname{Ex} 1.1$}

$$
\begin{aligned}
& u(x, 0, \xi)=\left\{\begin{array}{ll}
u_{L}=1+\hat{\sigma} \xi & x<x_{0} \\
u_{R}=-1-\hat{\sigma} \xi & x<x_{0}
\end{array} u(x, 0, \xi)= \begin{cases}u_{L}=1+\hat{\sigma} \xi & x<x_{0} \\
u_{R}=-1+\hat{\sigma} \xi & x<x_{0}\end{cases} \right. \\
& u(x, 0)=\cos (\pi x)(1+\hat{\sigma} \xi) \quad u(x, 0)=\cos (\pi x)+\hat{\sigma} \xi \\
& \xi \in N(0,1) \quad \xi \in N(0,1) \\
& \rho_{L R}=-1 \quad \rho_{L R}=1
\end{aligned}
$$

The problems are similar in terms of expected value and variance at the boundary, but the difference in correlation between the left and right states completely change the behavior over time. The difference in initial variance in the interior of the domain has only a limited impact on the time dependent difference between the solutions; this has been checked by varying the initial functions. Note that Ex 1.1 is included to show the importance of the sign of the stochastic variable, but is a special case of a more general phenomenon of superimposition of discontinuities exhibited by Ex 1.2 and further explained and analyzed below. Figure 5 shows the two cases at time $t=0.5$ for $M=3$. We use $\hat{\sigma}=0.1$ and $x_{0}=0.5$. 

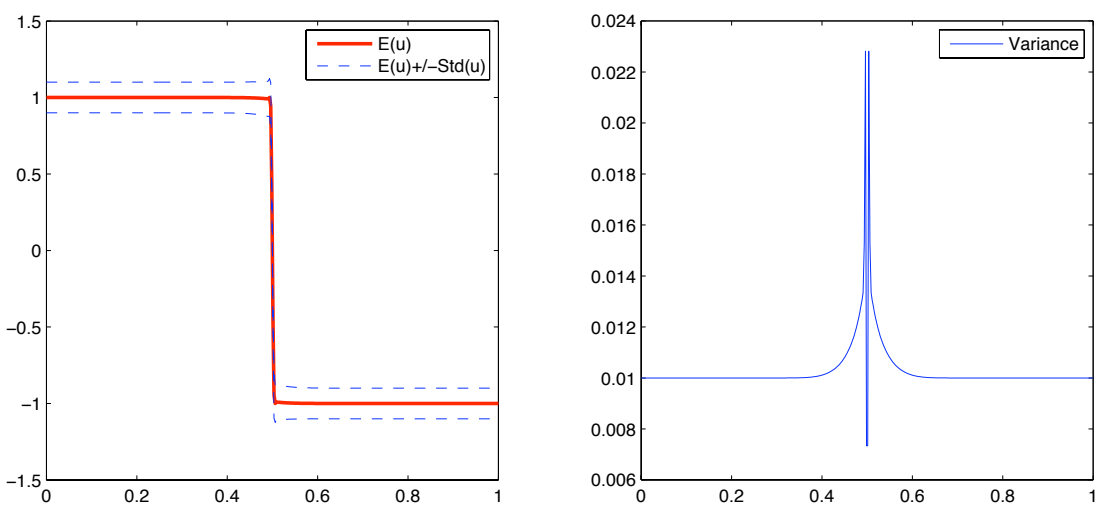

(a) Ex 1.1. Symmetric boundary conditions.
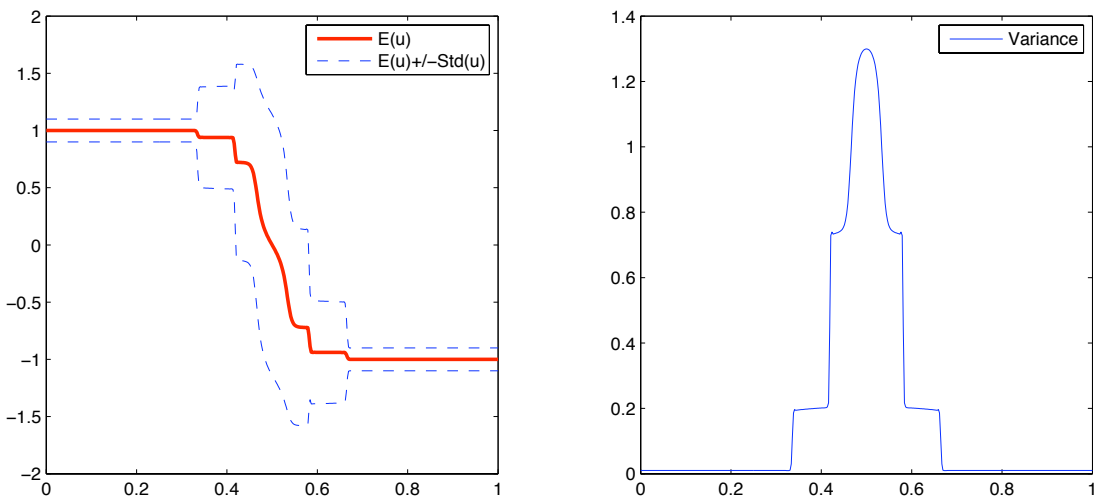

(b) Ex 1.2. Constant initial variance.

Figure 5: Development of variance of the perturbed cosine wave. $t=0.5$ for $M=3$, $m=400$.

To explain the differences between the solutions depicted in Figure 5, we turn to analysis of the characteristics for the truncated system with $M=1$. The polynomial chaos coefficients of the boundaries are given by 


$$
\left.\left.\begin{array}{l}
u_{0}=1 \\
u_{1}=0.1
\end{array}\right\} x=0, \quad \begin{array}{l}
u_{0}=-1 \\
u_{1}=-0.1
\end{array}\right\} x=1
$$

and

$$
\left.\left.\begin{array}{l}
u_{0}=1 \\
u_{1}=0.1
\end{array}\right\} x=0, \quad \begin{array}{l}
u_{0}=-1 \\
u_{1}=0.1
\end{array}\right\} x=1
$$

respectively.

Note that with more polynomial chaos coefficients included, the higher order coefficients are zero at the boundaries. The expected boundary values as well as the boundary variance are the same for Ex 1.1 and Ex 1.2. In order to relate the concepts of characteristics with expected value and variance, we will use the fact that the expected value at each point is the average of the characteristics,

$$
\mathrm{E}(u)=u_{0}=\frac{w_{1}+w_{2}}{2}
$$

and that the variance depends on the distance between the characteristics,

$$
\operatorname{Var}(u)=u_{1}^{2}=\left(\frac{w_{1}-w_{2}}{2}\right)^{2} .
$$

To explain the qualitative differences between the two cases Ex 1.1 and Ex 1.2 , consider the decoupled system (52). The boundary values for $u_{0}$ and $u_{1}$ are inserted into the characteristic variables $w_{1}$ and $w_{2}$; discontinuities emerge when the characteristics meet.

For Ex 1.1 we have $w_{1}(x=0)=-w_{1}(x=1)$ and $w_{2}(x=0)=-w_{2}(x=$ 1). Inserting these values in (53) and (54) gives the shock speeds $s_{w_{1}}=s_{w_{2}}=$ 0 , corresponding to two stationary shocks (of different magnitude) at $x=0.5$, which can be seen in Figure 6a. Inserting the characteristic values (can be 
evaluated in Figure 6) into Eq. (59) result in uniform variance except around the discontinuity, Figure 7a. Since the characteristic solution is propagating from the boundaries, this interval shrinks with time and collapses at $x=0.5$.

In Ex. 1.2, the characteristics are $w_{1}(x=0)=1.1>-w_{1}(x=1)=0.9$ and $w_{2}(x=0)=0.9<-w_{2}(x=1)=1.1$. Evaluating (53) and (54) when the characteristics cross yields $s_{w_{1}}=0.1$ and $s_{w_{2}}=-0.1$. The discontinuity when the characteristics meet will then split and propagate as two moving shocks in $u_{0}$ and $u_{1}$, located equidistantly from the mid-point $x=0.5$. In $w_{1}$ and $w_{2}$ there will still be a single shock. The shock speeds are given by the expressions (53)-(56). The vertical gap between the characteristics at $x=0.5$ in Figure $6 \mathrm{~b}$ corresponds to the variance peak at this location in $5 \mathrm{~b}$.
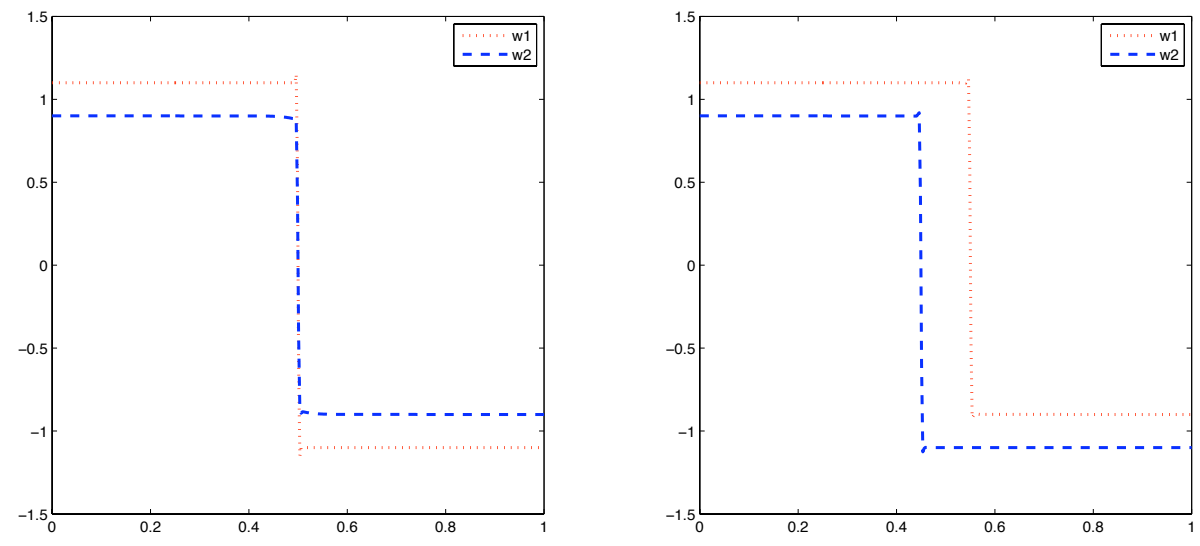

(a) Ex 1.1. The variance is undefined at (b) Ex 1.2. The variance peaks at $x=0.5$. $x=0.5$. $w_{1}$ is left-going and $w_{2}$ is right-going.

Figure 6: Characteristics of the two perturbed cosine waves (Ex 1.1 and Ex 1.2) for $M=1$. 

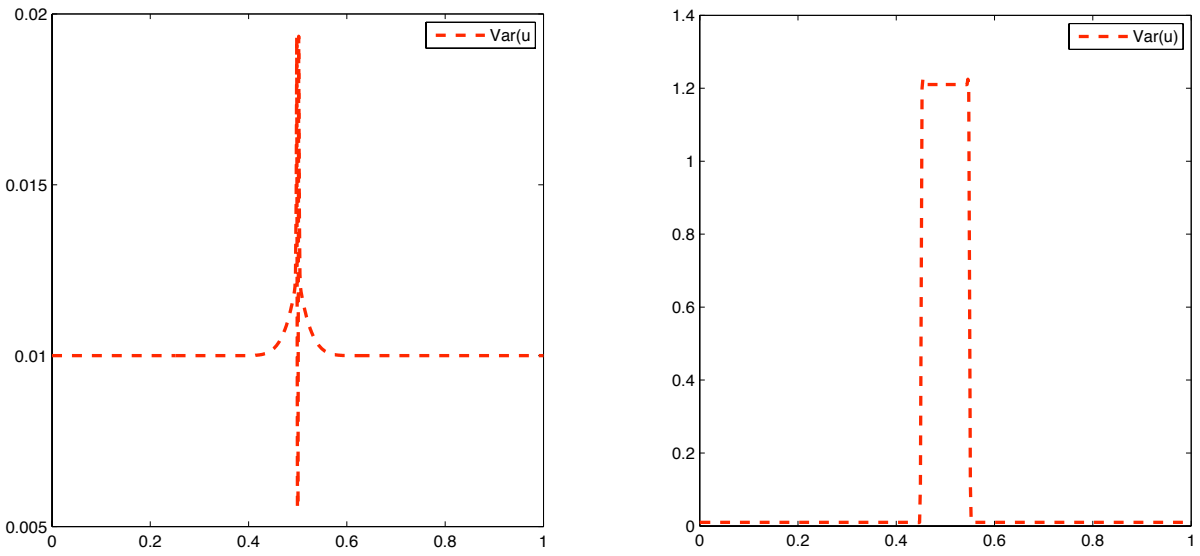

(a) Ex 1.1. The variance is constant ex- (b) Ex 1.2. The variance is maximal at cept around the discontinuity. the shock location and spreads towards the boundaries.

Figure 7: Variance of Ex. 1.1 and Ex. 1.2 for $M=1$, calculated from $w_{1}, w_{2}$ using (59).

The system used for analysis of characteristics is truncated to $M=1$, but the conclusions about the qualitative behavior holds for higher order systems. Including more polynomial chaos coefficients would result in additional shocks of different magnitude and speed. Observe the qualitative similarities between the solutions in Figure 6 and Figure 5. Regardless of the truncation of polynomial chaos coefficients, the variance approaches 0 at the shock location in Ex 1.1. At the shock location in Ex 1.2 the variance reaches a maximum that will spread towards the boundaries and cancel the discontinuity. The observation that the same boundary and initial expected value and variance can give totally different solutions indicates that knowledge about the polynomial chaos coefficients is required to obtain a unique solution. 
The analysis of characteristics further shows that the problem could be partitioned into several phases of development, depending on the speeds of the characteristics. Consider again the boundary conditions of Ex 1.1 and Ex 1.2 but now assume $u(x, 0)=0$ for $x \in(0,1)$. The solution for $M=1$ before the characteristics meet is shown in Figure 8. With more polynomial chaos coefficients, the sharp edges in the solution will disappear. At time $t=0.5$, the solutions to the two problems are still similar, with two variance peaks at the shocks that are traveling towards the middle of the domain.
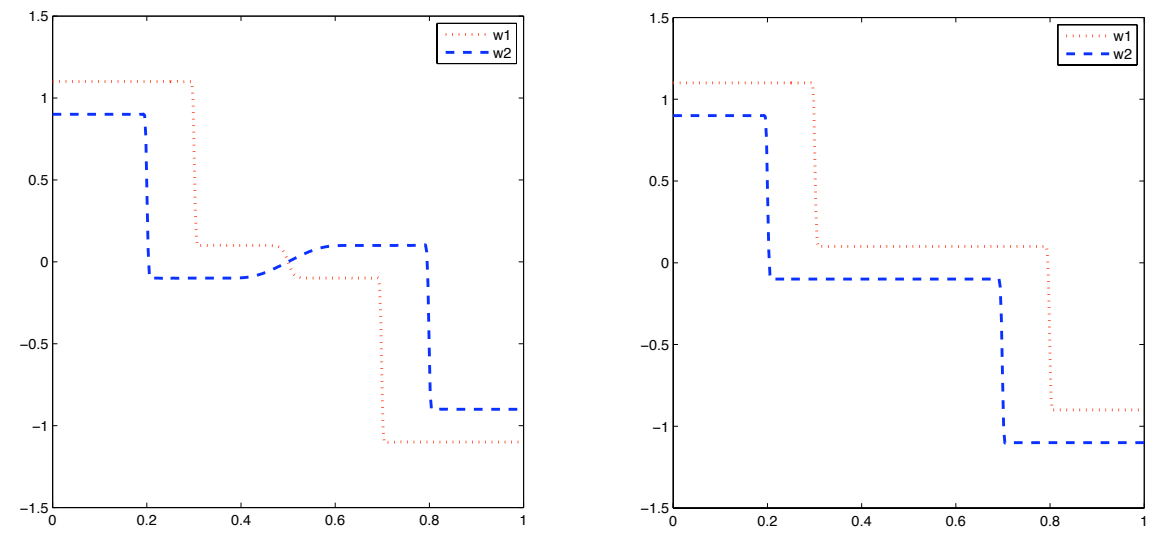

(a) Ex 1.1. Boundary conditions: (b) Ex 1.2. Boundary conditions:

$$
\begin{aligned}
u(0, t)=(1,0.1,0, \ldots) ; \quad u(1, t)= & u(0, t)=(1,0.1,0, \ldots) ; u(1, t)= \\
(-1,-0.1,0, \ldots) . & (-1,0.1,0, \ldots) .
\end{aligned}
$$

Figure 8: Characteristics at $t=0.5, M=1$.

For comparison, Figure 9 shows the expected value and variance calculated from the characteristics in Figure 8. 

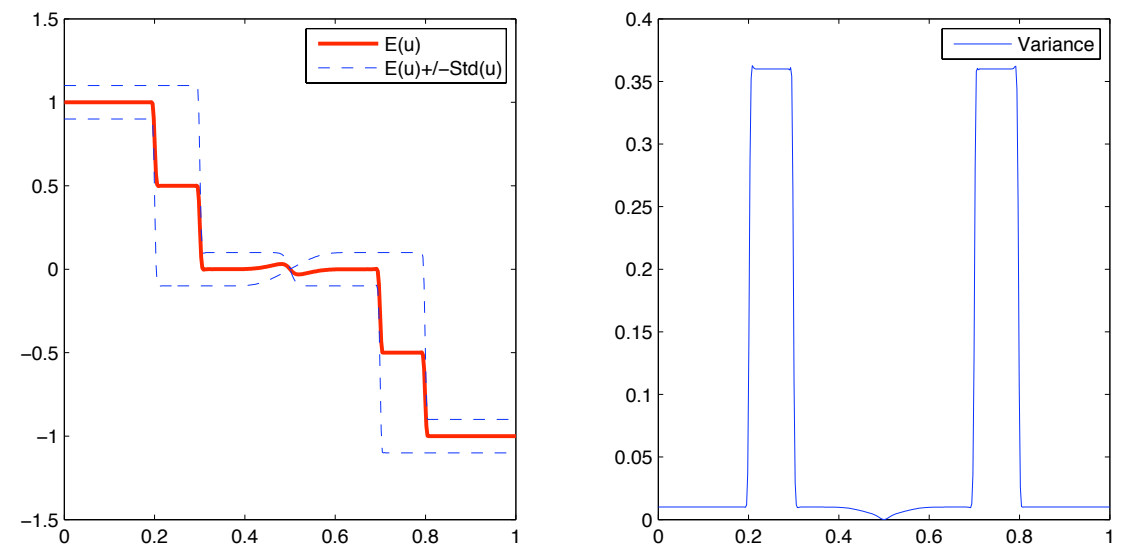

(a) Ex 1.1. Symmetric boundary conditions: $u(0, t)=(1,0.1,0, \ldots) ; u(1, t)=$ $(-1,-0.1,0, \ldots)$.
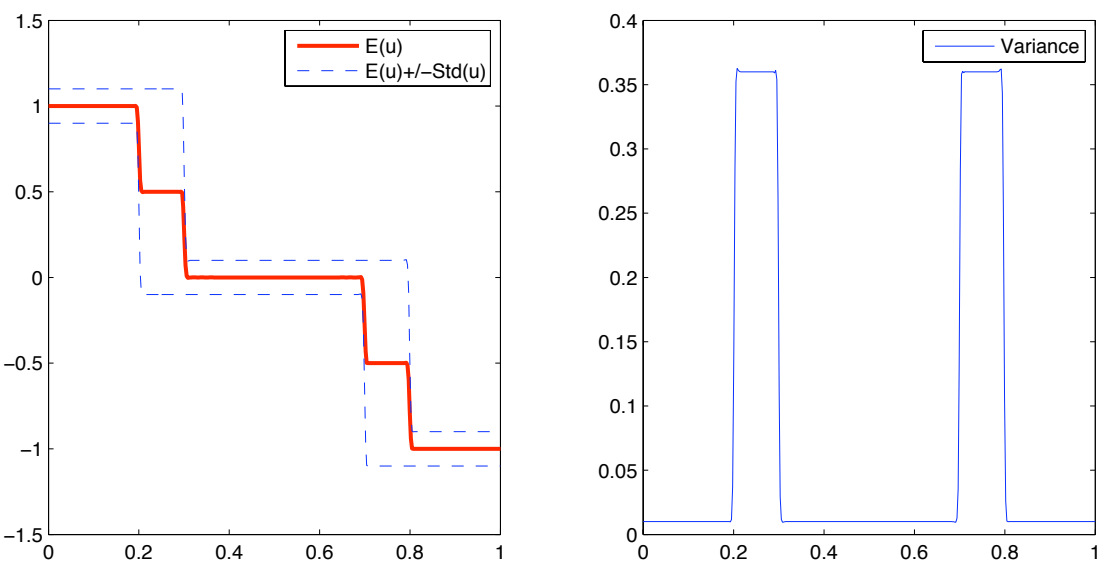

(b) Ex. 1.2. Boundary conditions: $u(0, t)=(1,0.1,0, \ldots) ; u(1, t)=(-1,0.1,0, \ldots)$.

Figure 9: Expected value and variance at $t=0.5, M=1$.

Asymptotically in time, the symmetric problem (Ex. 1.1) will result in a stationary shock. The variance will equal the initial boundary variance 
except for a peak at the very location of the shock. The boundary conditions are independent of time. This property is illustrated in Figure 10a, where the solution has reached steady state.

The time development of the solution of Ex 1.2 is not consistent with the stationary boundary conditions stated in the problem formulation. The characteristics are transported from one boundary to the other (see Figure 10b), thus changing the boundary data. The boundary conditions of Ex 1.2 must therefore be time-dependent (and can be calculated exactly from (14) for this example). Unlike the continuously varying boundary conditions of the full polynomial chaos expansion problem, the boundary conditions for the truncated system of Figure 10b will change discontinuosly from the initial boundary condition to zero at the moment the characteristics reach the boundaries. In a general hyperbolic problem, the imposition of correct time-dependent boundary conditions might become one of the more significant problems with the PC method. A detailed investigation is necessary to identify an approach to specify time-dependent stochastic boundary data, especially for the higher order moments. Special non-reflecting boundary conditions will be required. In the case studied here, analytical boundary conditions have been derived and can be correctly imposed for any time and order of chaos expansions. 

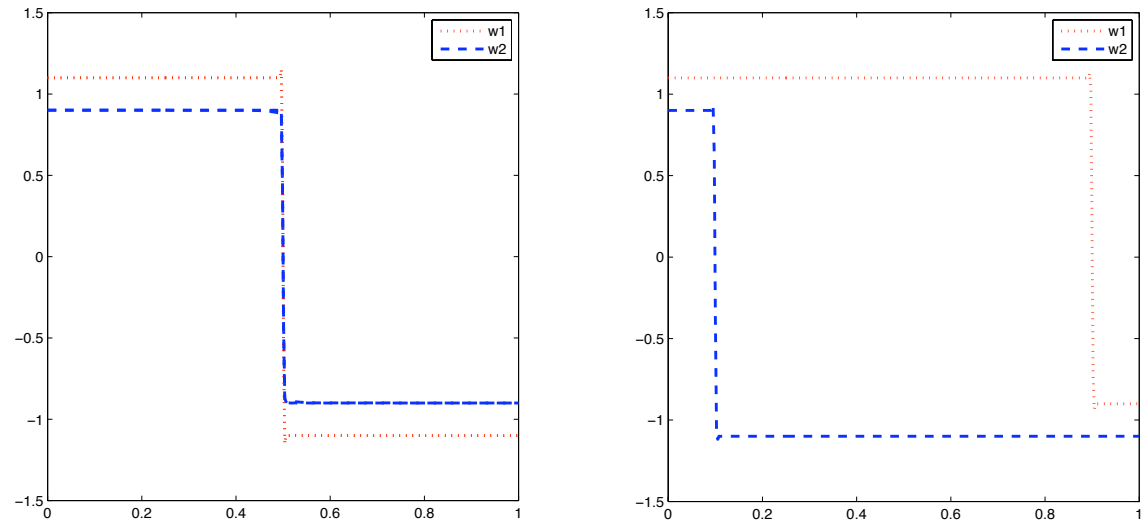

(a) Ex 1.1. Characteristics have reached (b) Ex 1.2. $w_{1}$ is right-going, $w_{2}$ leftsteady-state. going.

Figure 10: Characteristics at $t=4$ for $M=1$.

\section{Summary and conclusions}

The polynomial chaos approach, together with finite difference methods, is used to solve the Burgers' equation under uncertain initial and boundary conditions. Stable difference schemes are obtained by the use of artificial dissipation, difference operators satisfying the summation by parts property and a weak imposition of characteristic boundary conditions.

A number of mathematical properties of the deterministic Burgers' equation hold for the hyperbolic problem that results from the Galerkin projection of the truncated PC expansions. The system is symmetric and a split form combining conservative and non-conservative formulations is used to obtain an energy estimate. The truncated linearized problem is shown to be wellposed. The system eigenvalues cannot be computed analytically and this makes the choice of the time step difficult; moreover, this affects the accu- 
racy of the methods since the dissipation operators are eigenvalue dependent. An eigenvalue estimate is provided.

Even though the solution of the Burgers' equation is discontinuous for a particular value of the uncertain (stochastic) variable, the polynomial chaos coefficient functions are in general continuous for the Riemann problems investigated. The solution coefficients of the truncated system are discontinuous and can be treated as a superimposition of a finite number of discontinuous characteristic variables. This has been shown explicitly for the $2 \times 2$-case. The discontinuous coefficients converge with the number of polynomial chaos coefficients to continuous functions.

Examples have shown the need to provide time-dependent boundary conditions that might include higher order moments. Stochastic time-dependent boundary conditions have been derived for the Burgers' equation.

[1] Guide for the Verification and Validation of Computational Fluid Dynamics Simulations. Number AIAA-G-077-1998. American Institute of Aeronautics and Astronautics, Reston, VA, 1998.

[2] P.S. Beran, C.L. Pettit, D.R. Millman, Uncertainty quantification of limit-cycle oscillations, Journal of Computational Physics 217(1) (2006) $217-247$.

[3] R.H. Cameron, W.T. Martin, The Orthogonal Development of NonLinear Functionals in Series of Fourier-Hermite Functionals, The Annals of Mathematics 48(2) (1947) 385-392.

[4] M. H. Carpenter, D. Gottlieb, S. Abarbanel, Time-stable boundary conditions for finite-difference schemes solving hyperbolic systems: method- 
ology and application to high-order compact schemes, Journal of Computational Physics 111 (1994) 220-236.

[5] M.H. Carpenter, J. Nordström, D. Gottlieb, A stable and conservative interface treatment of arbitrary spatial accuracy, Journal of Computational Physics 148 (1999) 341-365.

[6] C. Chauvière, J.S. Hesthaven, L. Lurati, Computational Modeling of Uncertainty in Time-Domain Electromagnetics, SIAM J. Sci. Comput. 28(2) (2006) 751-775.

[7] Q.-Y. Chen, D. Gottlieb, J.S. Hesthaven, Uncertainty analysis for the steady-state flows in a dual throat nozzle, Journal of Computional Physics 204 (2005) 378-398.

[8] M. Christie, V. Demyanov, D. Erbas, Uncertainty quantification for porous media flows, Journal of Computational Physics 217(1) (2006) $143-158$.

[9] D. Funaro, Polynomial approximation of differential equations, SpringerVerlag, New York, 1992.

[10] R. Ghanem, A. Doostan, J. Red-Horse, A probabilistic construction of model validation, Computer Methods in Applied Mechanics and Engineering 197 (2008) 2585-2595.

[11] G.H. Golub and C.F. van Loan, Matrix Computations, second ed., Johns Hopkins University Press, Baltimore, 1989. 
[12] D. Gottlieb, J.S. Hesthaven, Spectral methods for hyperbolic problems, J. Comput. Appl. Math. 128(1-2) (2001) 83-131.

[13] B. Gustafsson, H.-O. Kreiss and J. Oliger, Time dependent problems and difference methods, Wiley, 1995.

[14] T.Y. Hou, W. Luo, B. Rozovskii, H.-M. Zhou, Wiener Chaos expansions and numerical solutions of randomly forced equations of fluid mechanics, Journal of Computational Physics 216(2) (2006) 687-706.

[15] R. LeVeque, Numerical Methods for Conservation Laws, second ed., Birkh?user, Basel, 2006.

[16] K. Mattsson, M. Svärd, J. Nordström, Stable and Accurate Artificial Dissipation, Journal of Scientific Computing 21(1) (2004) 57-79.

[17] J. Nordström, Conservative Finite Difference Formulations, Variable Coefficients, Energy Estimates and Artificial Dissipation, Journal of Scientific Computing 29(3) (2006) 375-404.

[18] J. Nordström, M.H. Carpenter, Boundary and interface conditions for high-order finite-difference methods applied to the Euler and NavierStokes equations, Journal of Computational Physics 148 (1999) 621-645.

[19] J. Nordström, M.H. Carpenter, High-order finite difference methods, multidimensional linear problems, and curvilinear coordinates, Journal of Computational Physics 173 (2001) 149-174.

[20] S.V. Poroseva, J. Letschert, M. Y. Hussaini, Application of Evidence Theory to Quantify Uncertainty in Forecast of Hurricane Path, Pro- 
ceed. of the 18th Conference on Probability and Statistics, the American Meteorological Society 86th Annual Meeting, Atlanta (GA), 2006

[21] M.T. Reagan, H.N. Najm, R.G. Ghanem, O.M. Knio, Uncertainty quantification in reacting-flow simulations through non-intrusive spectral projection, Combustion and Flame 132(3) (2003) 545-555.

[22] R.D. Richtmyer, K.W. Morton, Difference Methods for Initial-Value Problems, second ed., Interscience Publishers, New York, 1967.

[23] B. Strand, Summation by parts for finite difference approximations for d/dx, Journal of Computational Physics 110 (1994) 47-67.

[24] G. Szegö Orthogonal Polynomials, fourth ed., American Mathematical Society, Providence, 1975.

[25] X. Wan, G.E. Karniadakis, Long-term behavior of polynomial chaos in stochastic flow simulations, Computer methods in applied mechanics and engineering 195(41-43) (2006) 5582-5596.

[26] N. Wiener, The Homogeneous Chaos, American Journal of Mathematics 60(4) (1938) 897-936.

[27] J. Witteveen, S. Sarkar, H. Bijl, Modeling physical uncertainties in dynamic stall induced fluid-structure interaction of turbine blades using arbitrary polynomial chaos, Comput. Struct. 85 (2007) 866-878.

[28] D. Xiu, G.E. Karniadakis, The Wiener-Askey Polynomial Chaos for Stochastic Differential Equations, SIAM J. Sci. Comput. 24(2) (2002) 619-644. 
[29] D. Xiu, G.E. Karniadakis, Modeling uncertainty in flow simulations via generalized polynomial chaos, Journal of Computational Physics 187(1) (2003) 137-167.

[30] D. Xiu and G.E. Karniadakis, Supersensitivity due to uncertain boundary conditions, International Journal for Numerical Methods in Engineering 61 (2004) 2114-2138.

[31] Y. Yu, M. Zhao, T. Lee, N. Pestieau, W. Bo, J. Glimm, J. W. Grove, Uncertainty quantification for chaotic computational fluid dynamics, Journal of Computational Physics 217(1) (2006) 200-216. 